\title{
A unified energy formulation for the stability analysis of open and closed thin-walled members in the framework of the generalized beam theory
}

\author{
P. Simão, L. Simões da Silva* \\ Department of Civil Engineering, University of Coimbra, Polo II, Pinhal de Marrocos, \\ 3030 Coimbra, Portugal
}

Received 15 January 2003; received in revised form 17 February 2004; accepted 17 February 2004

\begin{abstract}
Generalized beam theory-GBT - is among the most adequate tools for the analysis of thin-walled prismatic elements. It enables the analysis of the distortion of the element crosssection and local buckling of individual walls in a unified manner that incorporates the results from classical bending theory. The basis of this theory was developed in the 1960s by Schardt for first and second order elastic behaviour of thin-walled members.

Open and closed thin-walled members present the distinctive difference of the unknown shear flow that characterizes the latter. More specifically, shear strains must follow an elasticity law, as opposed to the simplifying assumptions for open cross-sections.

It is the purpose of the present paper to present a unified energy formulation for the nonlinear analysis of both open and closed sections in the framework of GBT, able to deal with all modal interaction phenomena between local plate behaviour, distortional behaviour and the more classical global (flexural, torsional and flexural-torsional) response. Finally, an application to the stability analysis of a compressed thin-walled column is presented and discussed.
\end{abstract}

(C) 2004 Elsevier Ltd. All rights reserved.

Keywords: Thin-walled members; Generalized beam theory; Local buckling; Distortional buckling; Mode interaction

\footnotetext{
${ }^{*}$ Corresponding author. Tel.: +351-239-797-216; fax: +351-239-797-217.

E-mail address: luis_silva@gipac.pt (L.S. da Silva).
} 


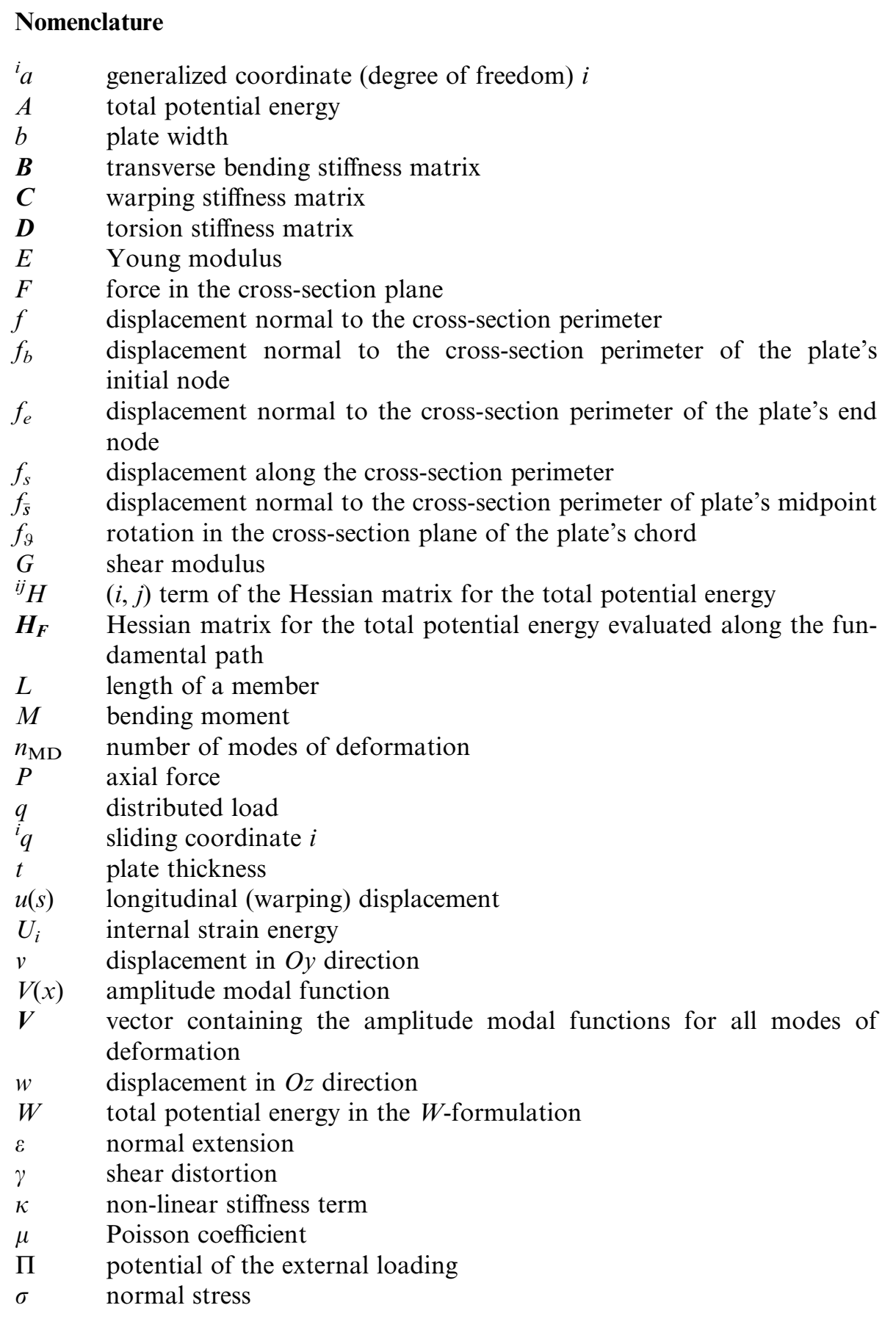


$\tau \quad$ shear stress

( ) denotes differentiation along the perimeter coordinate $s$

()$^{\prime}$ denotes differentiation along the longitudinal coordinate $x$

ij ()$\quad(i, j)$ term of a matrix

${ }^{i}() \quad$ denotes mode of deformation $i$

()$_{\sigma}$ indicates non-linear term related to the normal longitudinal membrane stresses

()$_{S H}$ indicates non-linear term related to shear membrane stresses

\section{Introduction}

\subsection{Generalities}

In recent years, the use of very slender thin-walled cross-section members has become increasingly common, due to its high stiffness/weight ratio. Extensive application of these members is found, in practice, in cold-formed members for lightweight structures [1,2] or in box girder bridges [3]. The high slenderness that characterizes these members implies a great susceptibility to various instability phenomena [4]. In fact, in addition to the usual global instability phenomena (flexural, torsional or flexural-torsional buckling or lateral torsional buckling), related to the deformation of the member axis combined exclusively with rigid-body displacement of the cross-sections, distortional and local plate instability phenomena are now a potential problem.

Thin-walled closed cross-section members present the distinctive feature of a higher torsional stiffness when compared to their open cross-section counterparts. This increase in torsional stiffness is associated with a constant shear flow around the cross-section that does not exist in open sections subjected to torsion. To deal with this additional complexity, the theory of thin-walled closed section members, as developed by von Kármán and Christensen [5], relaxes the well-known Vlasov's [6] assumption of negligible membrane shear distortion. Classically [5], the usual strategy to determine this statically indeterminate shear flow is to consider the existence of two shear flows: the main shear flow obtained through a constitutive relation, and a secondary shear flow obtained by equilibrium, considering the variation along the length of the membrane longitudinal normal stresses, which is, usually, much smaller than the first one and will be neglected in setting up the global member equilibrium.

Generalized beam theory - GBT - is a whole theory devoted to the analysis of thin-walled prismatic members, developed since the 1960s by Schardt and his co-workers [4,7-11] at the Technical University of Darmstadt, in Germany, and has been widely applied to study the behaviour of cold formed members $[1,12,13]$. It can be regarded as a fusion between the classical Vlasov's theory for thin-walled 
members [6] and the folded plate theory [14-16], and is an alternative to the classical finite strip [3] and finite element methods. It enables the analysis of thinwalled prismatic members with the allowance of cross-section distortion and local plate behaviour, in a one-dimensional formulation through the linear combination of pre-established deformation patterns - the modes of deformation. Regarding the application of GBT for closed cross-sections, it is noted that an additional basic mode of deformation must be added to take into account the main shear flow around the cross-section, as well as its corresponding shear distortion, full details being given in [11].

It is the purpose of this paper to present a unified energy formulation for (open and closed) thin-walled sections that is able to deal with geometrical non-linear analysis, covering, in particular, bifurcational and post-bifurcational behaviour. More specifically, the following aspects are addressed in detail: (i) derivation of a common consistent energy formulation for open and closed cross-sections, (ii) highlight the differences between these two cross-sectional types and (iii) illustration of its application to the stability behaviour of open and closed section columns.

\subsection{The basic concepts of generalized beam theory}

The key concepts behind GBT consist of (i) the characterization of the behaviour of the prismatic members (evaluation of displacements and stresses) through a linear combination of predefined modes of deformation and (ii) algebraic diagonalization using an orthogonalization procedure of the modes of deformation to obtain the cross-sectional properties.

To illustrate the first aspect, the longitudinal displacements (warping functions) and the cross-section displacements normal to the walls are given by, respectively,

$$
\begin{aligned}
& u(x, s)=\sum_{k=1}^{n_{\mathrm{MD}}}{ }^{k} u(s) \cdot{ }^{k} V^{\prime}(x) \\
& f(x, s)=\sum_{k=1}^{n_{\mathrm{MD}}}{ }^{k} f(s) \cdot{ }^{k} V(x)
\end{aligned}
$$

where ${ }^{k} u(s)$ and ${ }^{k} f(s)$ denote the warping function and the cross-section displacements normal to the walls for mode of deformation $k$, respectively, and ${ }^{k} V(x)$ is the corresponding amplitude, $n_{\mathrm{MD}}$ being the total number of modes of deformatio$\mathrm{n}$. The remaining displacements, usually specified at the cross-sectional nodes, are described with similar expressions and related using kinematic compatibility relations, full details being found in [9].

To illustrate the second aspect, it is useful to recall Vlasov's theory for thin-walled prismatic members [6], where off-diagonal terms of the member equilibrium equations matrices become zero whenever the cross-section geometric properties are determined with reference to the cross-section principal coordinates. Since GBT is a generalization of Vlasov's theory, it is expectable that a similar phenomenon occurs. Starting with a set of $n_{\mathrm{MD}}$ linear equilibrium equations in each of the $n_{\mathrm{MD}}$ 
basic modes of deformation shown in Eqs. (1) and (2), generically represented by (terms related to the external loads not represented)

$$
\boldsymbol{C} \boldsymbol{V}^{\prime \prime \prime \prime}-\boldsymbol{D} \boldsymbol{V}^{\prime \prime}+\boldsymbol{B} \boldsymbol{V}=0,
$$

where $\boldsymbol{C}, \boldsymbol{D}$ and $\boldsymbol{B}$ represent the appropriate stiffness matrices [11], Schardt originally proposed an algebraic procedure to diagonalize matrices $\boldsymbol{C}, \boldsymbol{D}$ and $\boldsymbol{B}$ by establishing a set of three consecutive eigenvalue and eigenvector problems. In general, it is impossible to set into diagonal form all the three matrices but in certain cases, some off-diagonal terms may be neglected [11]. Also, diagonalizing two matrices of the equilibrium system will make the subsequent calculus much easier and gives physical meaning to the generalized geometric properties. After these three steps, all geometrical properties are obtained, making no resource of determining the centre of gravity, the principal axes and the shear centre of the cross-section. This gives elegance to the GBT procedure and also a mathematical meaning to the calculus of the geometrical properties through the cross-section principal coordinates in the classical theory.

\section{Energy formulation}

\subsection{Introduction and basic assumptions}

Consider a continuous, non-branched, thin-walled cross-section consisting of $n_{P P}$ main walls of longitudinally constant thickness $t_{p, r}$ and width $b_{p, r}$, rigidly connected at their end nodes, typically shown in Fig. 1. It is further assumed that two consecutive main walls make a non-zero continuity angle $\Delta \alpha_{i}$, so that the number of principal nodes is $n_{N P}=n_{P P}+1$ for an open section, and $n_{N P}=n_{P P}$ for a closed one. Following Schardt [11], and with reference to Fig. 1, two coordinate systems are defined: (i) a local coordinate system $\bar{s}_{r} s_{r} x$ for each wall $r$ and (ii) a global coordinate system, $x y z$ with its origin on node 1 . Displacements associated with global and local axes are defined in Table 1 and Fig. 2.

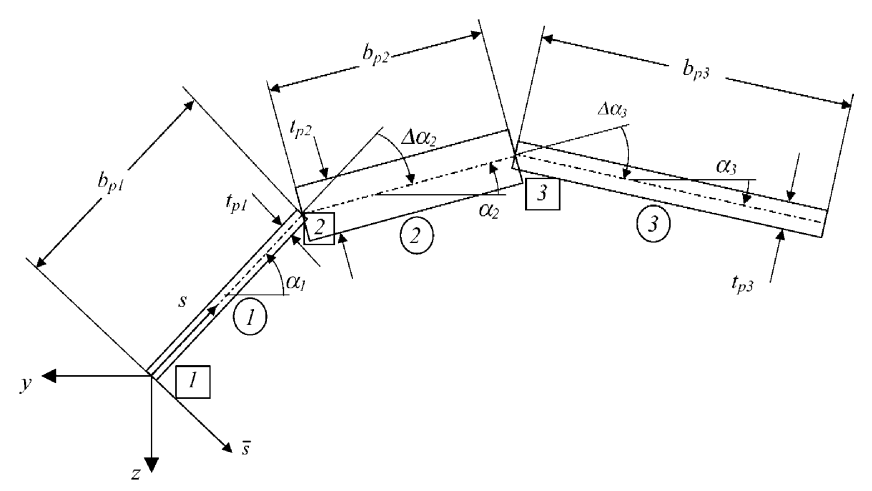

Fig. 1. Generic thin-walled cross-section. 
Table 1

Definition of the relevant displacements

\begin{tabular}{lllllll}
\hline & Axes & $\begin{array}{l}\text { Displace- } \\
\text { ment }\end{array}$ & $\begin{array}{l}\text { Displacement } \\
\text { of node } r\end{array}$ & $\begin{array}{l}\text { Displacement of } \\
\text { midpoint of wall } r\end{array}$ & \multicolumn{2}{c}{ Displacement of wall $r$} \\
\hline Global & $x$ & $u$ & $u_{r}$ & & & \\
axes & $y$ & $v$ & $v_{r}$ & & & \\
& $z$ & $w$ & $w_{r}$ & & $f_{b, r}$ \\
Local & $s$ & & & $f_{s, r}$ & \\
axes & $\bar{s}$ & $f$ & & $f_{\bar{s}, r}$ & & \\
& Rotation in & & & $f_{g, r}$ & & \\
& $O s \bar{s}$ plane & & & & & \\
\hline
\end{tabular}

Membrane and bending strains are denoted by

$$
\begin{aligned}
& \varepsilon_{x}=\varepsilon_{x}^{M}+\varepsilon_{x}^{B} \\
& \varepsilon_{s}=\varepsilon_{s}^{M}+\varepsilon_{s}^{B} \\
& \gamma=\gamma^{M}+\gamma^{B},
\end{aligned}
$$

while stresses and stress resultants are defined in Fig. 3.

In the framework of GBT, and in addition to the usual assumptions of elastic behaviour and small displacement plate bending behaviour of the cross-sectional walls, the following specific assumptions are considered [11]:

(i) negligible membrane transverse strain;

(ii) negligible membrane shear distortion, only for open cross-sections.

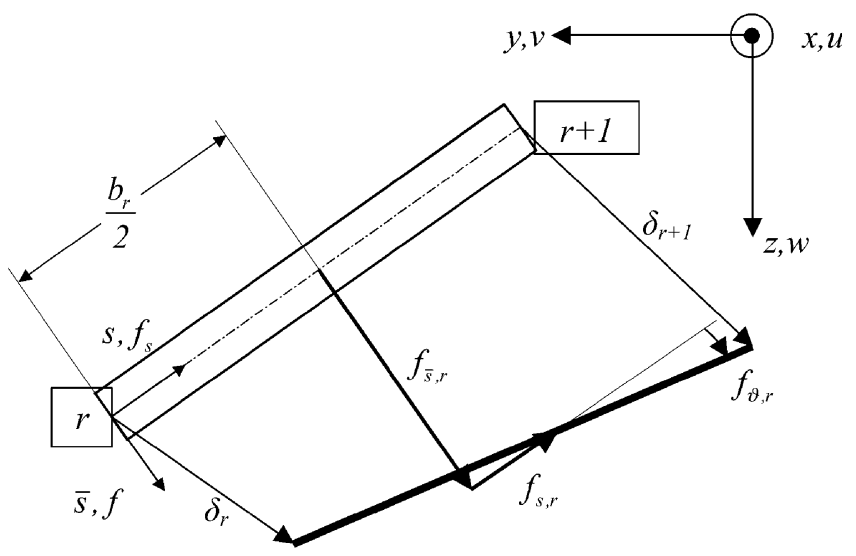

Fig. 2. Cross-section displacements in local coordinates. 

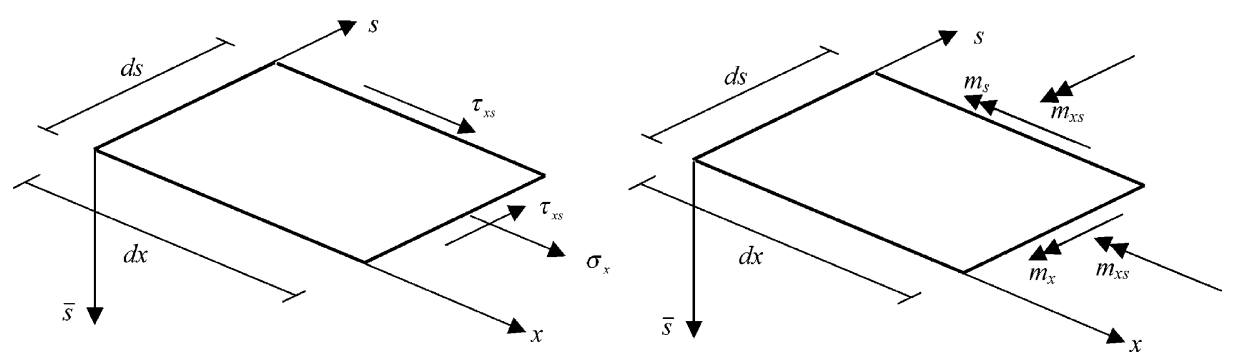

Fig. 3. Definition of stresses and bending stress resultants.

\subsection{Strain-displacement relations}

Using (') and ( $)^{\prime}$ to denote differentiation with respect to $s$ and $x$, respectively, the bending strain-displacement relations follow the usual linear formulation and are given by

$$
\begin{aligned}
& \varepsilon_{x}^{B}=-\bar{s} \frac{\partial^{2} f}{\partial x^{2}}=\sum_{k=1}^{n_{\mathrm{MD}}}-\bar{s}^{k} f^{k} V^{\prime \prime} \\
& \varepsilon_{s}^{B}=-\bar{s} \frac{\partial^{2} f}{\partial s^{2}}=\sum_{k=1}^{n_{\mathrm{MD}}}-\bar{s}^{k} \ddot{f}^{k} V \\
& \gamma_{s x}^{B}=-2 \bar{s} \frac{\partial^{2} f}{\partial s \partial x}=\sum_{k=1}^{n_{\mathrm{MD}}}-2 \bar{s}^{k} \dot{f}^{k} V^{\prime} .
\end{aligned}
$$

For a stability analysis, it is necessary to include the relevant non-linear terms in the membrane strain-displacement relations. From Fig. 4, the longitudinal

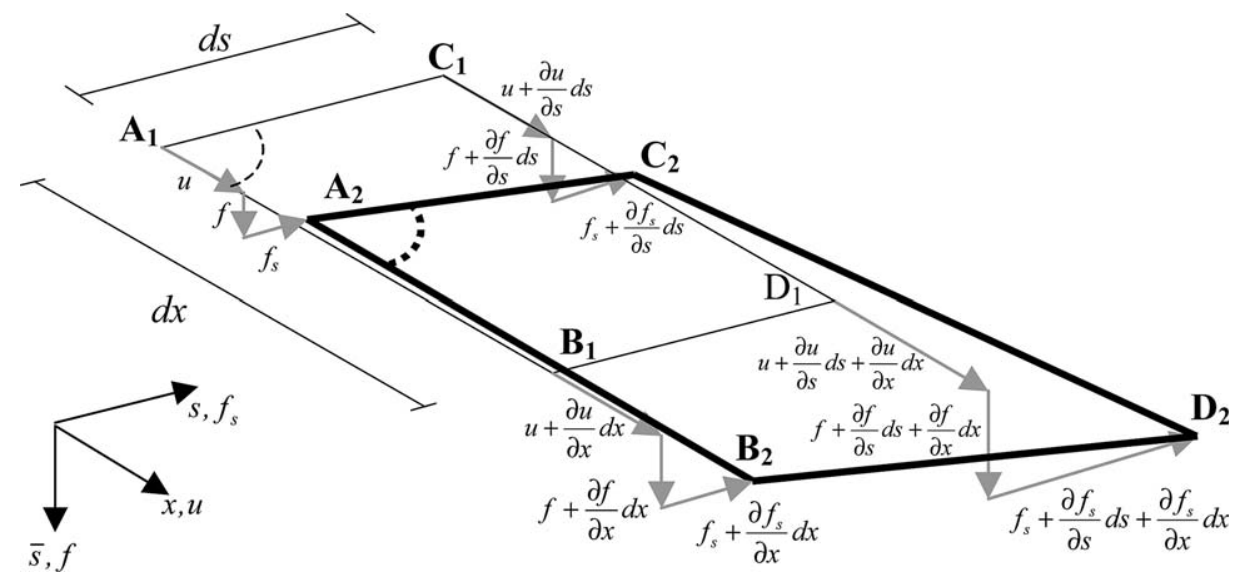

Fig. 4. Membrane displacements of the thin plate $\mathrm{d} s \times \mathrm{d} x$. 
membrane strain is given, in general, by:

$$
\varepsilon_{x}^{M}=\frac{\overline{A_{2} B_{2}}-\overline{A_{1} B_{1}}}{\overline{A_{1} B_{1}}}=\sqrt{\left(1+\frac{\partial u}{\partial x}\right)^{2}+\left(\frac{\partial f}{\partial x}\right)^{2}+\left(\frac{\partial f_{s}}{\partial x}\right)^{2}}-1
$$

Expanding Eq. (6) in Taylor series and neglecting higher-order terms yields:

$$
\begin{aligned}
\varepsilon_{x}^{M} & =\frac{\partial u}{\partial x}+\frac{1}{2}\left(\frac{\partial f}{\partial x}\right)^{2}+\frac{1}{2}\left(\frac{\partial f_{s}}{\partial x}\right)^{2} \\
& =\sum_{k=1}^{n_{\mathrm{MD}}}\left\{{ }^{k} u^{k} V^{\prime \prime}+\frac{1}{2} \sum_{l=1}^{n_{\mathrm{MD}}}\left[\left({ }^{k} f_{s}^{l} f_{s}+{ }^{k} f^{l} f\right)^{k} V^{\prime l} V^{\prime}\right]\right\} .
\end{aligned}
$$

Analogously, for closed cross-section members only, from the definition of membrane shear distortion,

$$
\gamma_{s x}^{M}=\triangleleft\left(B_{1} A_{1} C_{1}\right)-\triangleleft\left(B_{2} A_{2} C_{2}\right),
$$

given that

$$
\triangleleft\left(B_{1} A_{1} C_{1}\right)=\frac{\pi}{2} \mathrm{rad}
$$

and noting that by definition of scalar product of two vectors, $\overrightarrow{A_{2} B_{2}}$ and $\overrightarrow{A_{2} C_{2}}$, the angle $\triangleleft\left(B_{2} A_{2} C_{2}\right)$ may be determined as a function of the displacements of $A, B$ and $C$, the membrane shear distortion is given by:

$$
\gamma_{s x}^{M}=\frac{\pi}{2}-\arccos \left[\frac{\left(1+\frac{\partial u}{\partial x}\right) \frac{\partial u}{\partial s}+\left(1+\frac{\partial f_{s}}{\partial s}\right) \frac{\partial f_{s}}{\partial x}+\frac{\partial f}{\partial x} \frac{\partial f}{\partial s}}{\sqrt{\left(1+\frac{\partial u}{\partial x}\right)^{2}+\left(\frac{\partial f_{s}}{\partial x}\right)^{2}+\left(\frac{\partial f}{\partial x}\right)^{2}} \sqrt{\left(\frac{\partial u}{\partial s}\right)^{2}+\left(1+\frac{\partial f_{s}}{\partial s}\right)^{2}+\left(\frac{\partial f}{\partial s}\right)^{2}}}\right] .
$$

Expanding in Taylor series and neglecting higher-order terms leads to:

$$
\gamma_{s x}^{M}=\frac{\partial u}{\partial s}+\frac{\partial f_{s}}{\partial x}+\frac{\partial f}{\partial x} \frac{\partial f}{\partial s}+\frac{\partial u}{\partial x} \frac{\partial u}{\partial s}+\frac{\partial f_{s}}{\partial x} \frac{\partial f_{s}}{\partial s} .
$$

Finally, introducing the assumption of a negligible 1st order transverse membrane strain,

$$
\frac{\partial f_{s}}{\partial s} \approx 0
$$


the membrane shear distortion of Eq. (11) is obtained:

$$
\begin{aligned}
\gamma_{s x}^{M}= & \frac{\partial u}{\partial s}+\frac{\partial f_{s}}{\partial x}+\frac{\partial f}{\partial x} \frac{\partial f}{\partial s}+\frac{\partial u}{\partial x} \frac{\partial u}{\partial s}=\sum_{k=1}^{n_{\mathrm{MD}}}\left({ }^{k} \dot{u}+{ }^{k} f_{s}\right){ }^{k} V^{\prime}+\sum_{k=1}^{n_{\mathrm{MD}}} \sum_{l=1}^{n_{\mathrm{MD}}}{ }^{k} f^{l} \dot{f}^{k} V^{\prime l} V \\
& +\sum_{k=1}^{n_{\mathrm{MD}}} \sum_{l=1}^{n_{\mathrm{MD}}}{ }^{k} u^{l} \dot{u}^{k} V^{\prime \prime l} V^{\prime} .
\end{aligned}
$$

\subsection{Constitutive relations}

The stress--strain relations are split into bending effects,

$$
\begin{aligned}
& \sigma_{x}^{B}=\frac{E}{1-\mu^{2}}\left(\varepsilon_{x}^{B}+\mu \varepsilon_{x}^{B}\right)=\frac{E}{1-\mu^{2}} \sum_{i=1}^{n_{\mathrm{MD}}}-\bar{s}\left({ }^{i} f^{i} V^{\prime \prime}+\mu^{i} \ddot{f}^{i} V\right) \\
& \sigma_{s}^{B}=\frac{E}{1-\mu^{2}}\left(\varepsilon_{s}^{B}+\mu \varepsilon_{x}^{B}\right)=\frac{E}{1-\mu^{2}} \sum_{i=1}^{n_{\mathrm{MD}}}-\bar{s}\left(\ddot{f}^{i} V+\mu^{i} f^{i} V^{\prime \prime}\right) \\
& \tau_{s x}^{B}=G \gamma_{s x}^{B}=-2 G \sum_{i=1}^{n_{\mathrm{MD}}} \bar{s}^{i} \dot{f}^{i} V^{\prime} .
\end{aligned}
$$

and membrane effects (Eq. (15b) holds only for closed cross-section members):

$$
\begin{aligned}
& \sigma_{x}^{M}=E \varepsilon_{x}^{M}=E \sum_{i=1}^{n_{\mathrm{MD}}}\left\{{ }^{i} u^{i} V^{\prime \prime}+\frac{1}{2} \sum_{j=1}^{n_{\mathrm{MD}}}\left[\left({ }^{i} f_{s}^{j} f_{s}+{ }^{i} f^{j} f\right)^{i} V^{\prime j} V^{\prime}\right]\right\} \\
& \tau_{I}^{M}=G \gamma_{s x}^{M}=G\left[\sum_{i=1}^{n_{\mathrm{MD}}}\left({ }^{i} \dot{u}+{ }^{i} f_{s}\right)^{i} V^{\prime}+\sum_{i=1}^{n_{\mathrm{MD}}} \sum_{j=1}^{n_{\mathrm{MD}}}{ }^{i} \dot{f}^{j} V^{\prime j} V+\sum_{i=1}^{n_{\mathrm{MD}}} \sum_{j=1}^{n_{\mathrm{MD}}}{ }^{i} u^{j} \dot{u}^{i} V^{\prime \prime j} V^{\prime}\right] .
\end{aligned}
$$

\subsection{Strain energy}

From the classical definition of strain energy,

$$
U_{i}=\int_{0}^{L} \int_{A}\left(\frac{1}{2} \sigma_{x}^{M} \varepsilon_{x}^{M}+\frac{1}{2} \tau_{s x}^{M} \gamma_{s x}^{M}+\frac{1}{2} \sigma_{x}^{B} \varepsilon_{x}^{B}+\frac{1}{2} \sigma_{s}^{B} \varepsilon_{s}^{B}+\frac{1}{2} \tau_{s x}^{B} \gamma_{s x}^{B}\right) \mathrm{d} A \mathrm{~d} x,
$$

the bending terms are obtained by introducing Eqs. (5) and (14) into Eq. (16), yielding:

$$
\begin{aligned}
U_{i}^{B}= & \sum_{i=1}^{n_{\mathrm{MD}}} \sum_{k=1}^{n_{\mathrm{MD}}} \frac{1}{2} \int_{0}^{L}{ }^{L}{ }^{k} C^{B}{ }^{i} V^{\prime \prime k} V^{\prime \prime} \mathrm{d} x+\sum_{i=1}^{n_{\mathrm{MD}}} \sum_{k=1}^{n_{\mathrm{MD}}} \frac{1}{2} \int_{0}^{L}{ }^{i k} D_{1}{ }^{i} V^{\prime k} V^{\prime} \mathrm{d} x \\
& +\frac{1}{2} \sum_{i=1}^{n_{\mathrm{MD}}} \sum_{k=1}^{n_{\mathrm{MD}}} \int_{0}^{L}{ }^{L}{ }^{i k} D_{2}{ }^{i} V^{k} V^{\prime \prime} \mathrm{d} x+\frac{1}{2} \sum_{i=1}^{n_{\mathrm{MD}}} \sum_{k=1}^{n_{\mathrm{MD}}} \int_{0}^{L}{ }^{L}{ }^{i k} D_{2} T^{i} V^{\prime \prime k} V \mathrm{~d} x \\
& +\frac{1}{2} \sum_{i=1}^{n_{\mathrm{MD}}} \sum_{k=1}^{n_{\mathrm{MD}}} \int_{0}^{L}{ }^{L}{ }^{i k} B^{i} V^{k} V \mathrm{~d} x,
\end{aligned}
$$


where

$$
\begin{aligned}
& { }^{i k} C^{B}=\int_{s} \frac{E t^{3}}{12(1-\mu)} i^{i} f \mathrm{~d} s \\
& { }^{i k} D_{1}=\int_{s} \frac{G t^{3}}{3} i \dot{f}^{k} \dot{f} \mathrm{~d} s \\
& { }^{i k} D_{2}=\int_{s} \frac{E t^{3} \mu}{12(1-\mu)} i \ddot{f}^{k} f \mathrm{~d} s \\
& { }^{i k} D_{2 T}=\int_{s} \frac{E t^{3} \mu}{12(1-\mu)} i f^{k} \ddot{f} \mathrm{~d} s \\
& { }^{i k} B=\int_{s} \frac{E t^{3}}{12(1-\mu)} i \ddot{f}^{k} \ddot{f} \mathrm{~d} s
\end{aligned}
$$

Analogously, the membrane contribution, which includes all non-linear terms, is obtained by introducing Eqs. (7), (13) and (15) into Eq. (16), giving:

$$
\begin{aligned}
& U_{i}^{M}=\sum_{i=1}^{n_{\mathrm{MD}}} \sum_{k=1}^{n_{\mathrm{MD}}} \frac{1}{2} \int_{0}^{L}{ }^{i k} C^{M i} V^{\prime \prime k} V^{\prime \prime} \mathrm{d} x+\sum_{i=1}^{n_{\mathrm{MD}}} \sum_{k=1}^{n_{\mathrm{MD}}} \frac{1}{2} \int_{0}^{L}{ }^{i k} D_{3}{ }^{i} V^{\prime k} V^{\prime} \mathrm{d} x \\
& +\frac{1}{4} \sum_{i=1}^{n_{\mathrm{MD}}} \sum_{k=1}^{n_{\mathrm{MD}}} \sum_{l=1}^{n_{\mathrm{MD}}} \int_{L}{ }^{i k l} \kappa_{\sigma 2}{ }^{i} V^{\prime \prime k} V^{\prime l} V^{\prime} \mathrm{d} x+\frac{1}{4} \sum_{i=1}^{n_{\mathrm{MD}}} \sum_{j=1}^{n_{\mathrm{MD}}} \sum_{k=1}^{n_{\mathrm{MD}}} \int_{L}{ }^{i j k} \kappa_{\sigma 3}{ }^{i} V^{\prime j} V^{\prime k} V^{\prime \prime} \mathrm{d} x \\
& +\frac{1}{8} \sum_{i=1}^{n_{\mathrm{MD}}} \sum_{j=1}^{n_{\mathrm{MD}}} \sum_{k=1}^{n_{\mathrm{MD}}} \sum_{l=1}^{n_{\mathrm{MD}}} \int_{L}^{i j k l} \kappa_{\sigma 4}{ }^{i} V^{\prime j} V^{\prime k} V^{\prime l} V^{\prime} \mathrm{d} x+\frac{1}{2} \sum_{i=1}^{n_{\mathrm{MD}}} \sum_{k=1}^{n_{\mathrm{MD}}} \sum_{l=1}^{n_{\mathrm{MD}}} \int_{L}{ }^{i k l} \kappa_{S H 2}{ }^{i} V^{\prime k} V^{\prime l} V \mathrm{~d} x \\
& +\frac{1}{2} \sum_{i=1}^{n_{\mathrm{MD}}} \sum_{k=1}^{n_{\mathrm{MD}}} \sum_{l=1}^{n_{\mathrm{MD}}} \int_{L}{ }^{i k l} \kappa_{S H 3}{ }^{i} V^{\prime k} V^{\prime \prime l} V^{\prime} \mathrm{d} x+\frac{1}{2} \sum_{i=1}^{n_{\mathrm{MD}}} \sum_{j=1}^{n_{\mathrm{MD}}} \sum_{k=1}^{n_{\mathrm{MD}}} \int_{L}{ }^{i j k} \kappa_{S H 4}{ }^{i} V^{\prime j} V^{k} V^{\prime} \mathrm{d} x \\
& +\frac{1}{2} \sum_{i=1}^{n_{\mathrm{MD}}} \sum_{j=1}^{n_{\mathrm{MD}}} \sum_{k=1}^{n_{\mathrm{MD}}} \sum_{l=1}^{n_{\mathrm{MD}}} \int_{L}^{i j k l} \kappa_{S H 5}{ }^{i} V^{\prime j} V^{k} V^{\prime l} V \mathrm{~d} x \\
& +\frac{1}{2} \sum_{i=1}^{n_{\mathrm{MD}}} \sum_{j=1}^{n_{\mathrm{MD}}} \sum_{k=1}^{n_{\mathrm{MD}}} \sum_{l=1}^{n_{\mathrm{MD}}} \int_{L}^{i j k l} \kappa_{S H 6}{ }^{i} V^{\prime j} V^{k} V^{\prime \prime l} V^{\prime} \mathrm{d} x \\
& +\frac{1}{2} \sum_{i=1}^{n_{\mathrm{MD}}} \sum_{j=1}^{n_{\mathrm{MD}}} \sum_{k=1}^{n_{\mathrm{MD}}} \sum_{l=1}^{n_{\mathrm{MD}}} \int_{L}{ }^{i j k l} \kappa_{S H 7}{ }^{i} V^{\prime j} V^{k} V^{\prime l} V \mathrm{~d} x \\
& +\frac{1}{2} \sum_{i=1}^{n_{\mathrm{MD}}} \sum_{j=1}^{n_{\mathrm{MD}}} \sum_{k=1}^{n_{\mathrm{MD}}} \sum_{l=1}^{n_{\mathrm{MD}}} \int_{L}^{i j k l} \kappa_{S H 8}{ }^{i} V^{\prime j} V^{k} V^{\prime \prime l} V^{\prime} \mathrm{d} x \\
& +\frac{1}{2} \sum_{i=1}^{n_{\mathrm{MD}}} \sum_{j=1}^{n_{\mathrm{MD}}} \sum_{k=1}^{n_{\mathrm{MD}}} \sum_{l=1}^{n_{\mathrm{MD}}} \int_{L}{ }^{i j k l} \kappa_{S H 9}{ }^{i} V^{\prime \prime j} V^{\prime k} V^{\prime \prime l} V^{\prime} \mathrm{d} x,
\end{aligned}
$$


where the following coefficients relate to the longitudinal membrane stress

$$
\begin{aligned}
{ }^{i k} C^{M} & =\int_{s} E t^{i} u^{k} u \mathrm{~d} s \quad(1 \text { st order term }) \\
{ }^{i k l} \kappa_{\sigma 2} & =\int_{s} E t^{i} u\left({ }^{k} f_{s}^{l} f_{s}+{ }^{k} f^{l} f\right) \mathrm{d} s \\
{ }^{i j k} \kappa_{\sigma 3} & =\int_{s} E t^{k} u\left({ }^{i} f_{s}^{j} f_{s}+{ }^{i} f^{j} f\right) \mathrm{d} s \\
{ }^{i j k l} \kappa_{\sigma 4} & =\int_{s} E t\left({ }^{i} f_{s}^{j} f_{s}+{ }^{i} f^{j} f\right) \cdot\left({ }^{k} f_{s}^{l} f_{s}+{ }^{k} f^{l} f\right) \mathrm{d} s,
\end{aligned}
$$

while the following arise uniquely for closed sections from the membrane shear flow contribution:

$$
\begin{aligned}
& { }^{i k} D_{3}=\int_{s} G \cdot t \cdot\left({ }^{i} \dot{u}+{ }^{i} f_{s}\right) \cdot\left({ }^{k} \dot{u}+{ }^{k} f_{s}\right) \mathrm{d} s \text { (1st order term) } \\
& { }^{i k l} \kappa_{S H 2}=\int_{s} G \cdot t \cdot\left({ }^{i} \dot{u}+{ }^{i} f_{s}\right) \cdot{ }^{l} \dot{f} \cdot{ }^{k} f \mathrm{~d} s \\
& { }^{i k l} \kappa_{S H 3}=\int_{s} G \cdot t \cdot\left({ }^{i} \dot{u}+{ }^{i} f_{s}\right) \cdot{ }^{l} \dot{u} \cdot{ }^{k} u \mathrm{~d} s \\
& { }^{i j k} \kappa_{S H 4}=\int_{s} G \cdot t \cdot{ }^{i} f \cdot{ }^{j} \dot{f} \cdot\left({ }^{k} \dot{\boldsymbol{u}}+{ }^{k} f_{s}\right) \mathrm{d} s \\
& { }^{i j k l} \kappa_{S H 5}=\int_{s} G \cdot t \cdot{ }^{i} f \cdot{ }^{j} \dot{f} \cdot{ }^{l} \dot{f} \cdot{ }^{k} f \mathrm{~d} s \\
& { }^{i j k l} \kappa_{S H 6}=\int_{s} G \cdot t \cdot{ }^{i} f \cdot{ }^{j} \dot{f} \cdot{ }^{l} \dot{u} \cdot{ }^{k} u \mathrm{~d} s \\
& { }^{i j k} \kappa_{S H 7}=\int_{s} G \cdot t \cdot{ }^{i} u \cdot \cdot \dot{u} \cdot\left({ }^{k} \dot{u}+{ }^{k} f_{s}\right) \mathrm{d} s \\
& { }^{i j k l} \kappa_{S H 8}=\int_{s} G \cdot t \cdot{ }^{i}{ }^{\cdot j} \cdot{ }^{j} \cdot{ }^{l} \dot{f} \cdot{ }^{k} f \mathrm{~d} s \\
& { }^{i j k l} \kappa_{S H 9}=\int_{s} G \cdot t \cdot{ }^{i} u \cdot{ }^{j} \dot{u} \cdot{ }^{l} \dot{u} \cdot{ }^{k} u \mathrm{~d} s .
\end{aligned}
$$

\subsection{Energy potential of external loading}

The potential of the external loading is given, in general, by

$$
\begin{aligned}
\Pi= & \int_{L} \sum_{k=1}^{n_{\mathrm{MD}}} \sum_{r=1}^{n_{\mathrm{NT}}}\left\{q_{y, r}(x)^{k} v_{r}+q_{z, r}(x)^{k} w_{r}\right\}^{k} V \mathrm{~d} x+\int_{L} \sum_{k=1}^{n_{\mathrm{MD}}} \sum_{r=1}^{n_{\mathrm{NT}}}\left\{-q_{x, r}(x)^{k} u_{r}\right\}^{k} V^{\prime} \mathrm{d} x \\
& +P \times\left.{ }^{1} V^{\prime}\right|_{x=x_{1}}+\left.\sum_{k=2}^{n_{\mathrm{MD}}}\left({ }^{k} v_{\bar{r}} F_{y}+{ }^{k} w_{\bar{r}} F_{z}\right)^{k} V\right|_{x=x_{2}}+\left.{ }^{2} M^{2} V\right|_{x=x_{3}}+\left.{ }^{3} M^{3} V\right|_{x=x_{3}},(22)
\end{aligned}
$$


where $q_{y, r}(x), q_{z, r}(x)$ and $q_{x, r}(x)$ denote general longitudinally distributed loads applied at node $r$ in the $y, z$ and $x$ directions, respectively, $P$ denotes an axial force applied at $x=x_{1}, F$ is a concentrated force in the cross-section plane applied at $x=x_{2}$ and with vertical and horizontal components $F_{y}$ and $F_{z} \cdot{ }^{2} M$ and ${ }^{3} M$ denote the components of an applied bending moment at $x=x_{3}$ with respect to the principal cross-section axes.

\subsection{Total potential energy function}

Combining Eqs. (17), (19) and (22) yields the total potential energy function:

$$
\begin{aligned}
& \left.A=\sum_{i=1}^{n_{\mathrm{MD}}} \sum_{k=1}^{n_{\mathrm{MD}}} \frac{1}{2} \int_{0}^{L}{ }^{i k} C^{M}+{ }^{i k} C^{B}\right)^{i} V^{\prime \prime k} V^{\prime \prime} \mathrm{d} x \\
& +\sum_{i=1}^{n_{\mathrm{MD}}} \sum_{k=1}^{n_{\mathrm{MD}}} \frac{1}{2} \int_{0}^{L}\left({ }^{i k} D_{1}+{ }^{i k} D_{3}\right)^{i} V^{\prime k} V^{\prime} \mathrm{d} x+\frac{1}{2} \sum_{i=1}^{n_{\mathrm{MD}}} \sum_{k=1}^{n_{\mathrm{MD}}} \int_{0}^{L}{ }^{i k} D_{2}{ }^{i} V^{k} V^{\prime \prime} \mathrm{d} x \\
& +\frac{1}{2} \sum_{i=1}^{n_{\mathrm{MD}}} \sum_{k=1}^{n_{\mathrm{MD}}} \int_{0}^{L}{ }^{i k} D_{2 T^{i}} V^{\prime \prime k} V \mathrm{~d} x+\frac{1}{2} \sum_{i=1}^{n_{\mathrm{MD}}} \sum_{k=1}^{n_{\mathrm{MD}}} \int_{0}^{L}{ }^{L}{ }^{i k} B^{i} V^{k} V \mathrm{~d} x \\
& +\frac{1}{4} \sum_{i=1}^{n} \sum_{k=1}^{n_{\mathrm{MD}}} \sum_{l=1}^{n_{\mathrm{MD}}} \int_{L}{ }^{i k l}{ }^{{ }^{2}} \kappa_{\sigma 2}{ }^{i} V^{\prime \prime k} V^{\prime l} V^{\prime} \mathrm{d} x+\frac{1}{4} \sum_{i=1}^{n_{\mathrm{MD}}} \sum_{j=1}^{n_{\mathrm{MD}}} \sum_{k=1}^{n_{\mathrm{MD}}} \int_{L}{ }^{i j k} \kappa_{\sigma 3}{ }^{i} V^{\prime j} V^{\prime k} V^{\prime \prime} \mathrm{d} x \\
& +\frac{1}{8} \sum_{i=1}^{n_{\mathrm{MD}}} \sum_{j=1}^{n_{\mathrm{MD}}} \sum_{k=1}^{n_{\mathrm{MD}}} \sum_{l=1}^{n_{\mathrm{MD}}} \int_{L}^{i j k l} \kappa_{\sigma 4}{ }^{i} V^{\prime j} V^{\prime k} V^{\prime l} V^{\prime} \mathrm{d} x+\frac{1}{2} \sum_{i=1}^{n_{\mathrm{MD}}} \sum_{k=1}^{n_{\mathrm{MD}}} \sum_{l=1}^{n_{\mathrm{MD}}} \int_{L}{ }_{L}{ }^{i k l} \kappa_{S H 2}{ }^{i} V^{\prime k} V^{\prime l} V \mathrm{~d} x \\
& +\frac{1}{2} \sum_{i=1}^{n_{\mathrm{MD}}} \sum_{k=1}^{n_{\mathrm{MD}}} \sum_{l=1}^{n_{\mathrm{MD}}} \int_{L}^{i k l} \kappa_{S H 3}{ }^{i} V^{\prime k} V^{\prime \prime l} V^{\prime} \mathrm{d} x+\frac{1}{2} \sum_{i=1}^{n_{\mathrm{MD}}} \sum_{j=1}^{n_{\mathrm{MD}}} \sum_{k=1}^{n_{\mathrm{MD}}} \int_{L}{ }^{i j k} \kappa_{S H 4}{ }^{i} V^{\prime j} V^{k} V^{\prime} \mathrm{d} x \\
& +\frac{1}{2} \sum_{i=1}^{n_{\mathrm{MD}}} \sum_{j=1}^{n_{\mathrm{MD}}} \sum_{k=1}^{n_{\mathrm{MD}}} \sum_{l=1}^{n_{\mathrm{MD}}} \int_{L}^{i j k l} \kappa_{S H 5^{i}} V^{\prime j} V^{k} V^{\prime l} V \mathrm{~d} x \\
& +\frac{1}{2} \sum_{i=1}^{n_{\mathrm{MD}}} \sum_{j=1}^{n_{\mathrm{MD}}} \sum_{k=1}^{n_{\mathrm{MD}}} \sum_{l=1}^{n_{\mathrm{MD}}} \int_{L}^{i j k l} \kappa_{S H 6^{i}} V^{\prime j} V^{k} V^{\prime \prime l} V^{\prime} \mathrm{d} x \\
& +\frac{1}{2} \sum_{i=1}^{n_{\mathrm{MD}}} \sum_{j=1}^{n_{\mathrm{MD}}} \sum_{k=1}^{n_{\mathrm{MD}}} \int_{L}^{i j k} \kappa_{S H 7}{ }^{i} V^{\prime \prime j} V^{\prime k} V^{\prime} \mathrm{d} x+\frac{1}{2} \sum_{i=1}^{n_{\mathrm{MD}}} \sum_{j=1}^{n_{\mathrm{MD}}} \sum_{k=1}^{n_{\mathrm{MD}}} \sum_{l=1}^{n_{\mathrm{MD}}} \int_{L}^{i j k l} \kappa_{S H 8}{ }^{i} V^{\prime \prime j} V^{\prime k} V^{\prime l} V^{\prime} \mathrm{d} x \\
& +\frac{1}{2} \sum_{i=1}^{n_{\mathrm{MD}}} \sum_{j=1}^{n_{\mathrm{MD}}} \sum_{k=1}^{n_{\mathrm{MD}}} \sum_{l=1}^{n_{\mathrm{MD}}} \int_{L}^{i j k l} \kappa_{S H 9^{i}} V^{\prime \prime j} V^{\prime k} V^{\prime \prime l} V^{\prime} \mathrm{d} x \\
& -\int \sum_{k=1}^{n_{\mathrm{MD}}} \sum_{r=1}^{n_{\mathrm{NT}}}\left\{q_{y, r}(x)^{k} v_{r}+q_{z, r}(x)^{k} w_{r}\right\}^{k} V \mathrm{~d} x \\
& -\int \sum_{L=1}^{n_{\mathrm{MD}}} \sum_{r=1}^{n_{\mathrm{NT}}}\left\{-q_{x, r}(x)^{k} u_{r}\right\}^{k} V^{\prime} \mathrm{d} x-P \times\left.{ }^{1} V^{\prime}\right|_{x=x_{1}} \\
& -\left.\sum_{k=2}^{n_{\mathrm{MD}}}\left({ }^{k} v_{\bar{r}} F_{y}+{ }^{k} w_{\bar{r}} F_{z}\right)^{k} V\right|_{x=x_{2}}-\left.{ }^{2} M^{2} V\right|_{x=x_{3}}-\left.{ }^{3} M^{3} V\right|_{x=x_{3}} \text {. }
\end{aligned}
$$




\section{Application of the Rayleigh-Ritz method to compressed columns}

\subsection{Introduction}

In order to apply the general formulation derived above, the Rayleigh-Ritz method [17] will be used by approximating the modal amplitude functions ${ }^{k} V(x)$ by a set of coordinate functions ${ }^{k} V_{i}(x)$, as shown in Eq. (24):

$$
{ }^{k} V(x) \approx{ }^{k} a_{1}{ }^{k} V_{1}(x)+{ }^{k} a_{2}{ }^{k} V_{2}(x)+{ }^{k} a_{3}{ }^{k} V_{3}(x)+\ldots,
$$

where the coordinate functions ${ }^{k} V_{i}(x)$ must respect the kinematic boundary conditions and the coordinates ${ }^{k} a_{i}$ are the unknowns of the problem. Concentrating, in this paper, on an application to an open or closed simply supported compressed column with flexible end plates, a suitable and usual approximation consists of sinusoidal amplitude functions given by:

$$
\text { mode } 1: \quad{ }^{1} V(x) \approx{ }^{1} a \frac{x^{2} \sqrt{5}}{L^{5 / 2}}
$$

remaining modes: ${ }^{k} V(x) \approx{ }^{k} a \sin \frac{\pi x}{L} \quad$ for $k=2, \ldots, n_{\mathrm{MD}}$,

${ }^{1} a$ and ${ }^{k} a$ being the unknown degrees of freedom. It is noted that the approximation function for the first mode of deformation is not sinusoidal in order to allow for constant axial force along the length of the member. Note that other boundary conditions or different loading cases follow identical procedures, given an adequate choice of amplitude functions. For this particular case of a compressed column, the potential of the external loads is simply given by

$$
\Pi=P \times\left.{ }^{1} V^{\prime}\right|_{x=L},
$$

so that substitution of Eq. (25) into the total potential energy function (23) and integration along the length yields:

$$
\begin{aligned}
A= & A_{1}{ }^{1} a+A_{11}{ }^{1} a^{2}+\sum_{i=2}^{n_{\mathrm{MD}}} A_{i i}{ }^{i} a^{2}+\sum_{i=2}^{n_{\mathrm{MD}}} \sum_{j=2}^{n_{\mathrm{MD}}} A_{i j}{ }_{i j} a^{j} a+\sum_{i=2}^{n_{\mathrm{MD}}} \sum_{j=2}^{n_{\mathrm{MD}}}\left(A_{1 i j}{ }^{1} a^{i} a^{j} a+A_{11 i j}{ }^{1} a^{2 i} a^{j} a\right) \\
& +\sum_{i=2}^{n_{\mathrm{MD}}} \sum_{j=2}^{n_{\mathrm{MD}}} \sum_{k=2}^{n_{\mathrm{MD}}}\left(A_{i j k}{ }^{i} a^{j} a^{k} a+A_{1 i j k} a^{i} a^{j} a^{k} a\right)+\sum_{i=2}^{n_{\mathrm{MD}}} \sum_{j=2}^{n_{\mathrm{MD}}} \sum_{k=2}^{n_{\mathrm{MD}}} \sum_{l=2}^{n_{\mathrm{MD}}} A_{i j k l} a^{j} a^{j} a^{k} a
\end{aligned}
$$

where

$$
\begin{aligned}
& A_{1}=-P \frac{2 \sqrt{5}}{L^{3 / 2}} \\
& A_{11}={\frac{10}{L^{4}}}^{1} C
\end{aligned}
$$




$$
\begin{aligned}
& A_{i i}=\frac{\pi^{4}}{4 L^{3}}\left({ }^{i} C^{M}+{ }^{i} C^{B}\right)+\frac{L^{i}}{4} B+\frac{\pi^{2}}{4 L}\left({ }^{i i} D_{1}+{ }^{i i} D_{3}\right)-\frac{\pi^{2}}{4 L}\left({ }^{i i} D_{2}+{ }^{i i} D_{2 T}\right) \\
& A_{i j}=\frac{\pi^{2}}{4 L}\left({ }^{i j} D_{1}+{ }^{i j} D_{3}\right)-\frac{\pi^{2}}{4 L}\left({ }^{i j} D_{2}+{ }^{i j} D_{2 T}\right) \\
& A_{1 i j}=\frac{\pi^{2} \sqrt{5}}{4 L^{7 / 2}}\left({ }^{1 i j} \kappa_{\sigma 2}+{ }^{i j 1} \kappa_{\sigma 3}\right)-\frac{\pi^{2} \sqrt{5}}{2 L^{7 / 2}}\left({ }^{i j 1} \kappa_{S H 3}+{ }^{i 1 j} \kappa_{S H 7}\right) \\
& A_{i j k}=-\frac{\pi^{3}}{6 L^{3}}\left({ }^{i j k} \kappa_{\sigma 2}+{ }^{i j k} \kappa_{\sigma 3}\right)+\frac{\pi}{3 L}\left({ }^{i j k} \kappa_{S H 2}+{ }^{i j k} \kappa_{S H 4}\right) \\
& -\frac{\pi^{3}}{3 L^{3}}\left({ }^{i j k} \kappa_{S H 3}+{ }^{i j k} \kappa_{S H 7}\right) \\
& A_{11 i j}=\frac{5 \pi^{2}}{L^{6}}{ }^{1 i 1 j} \kappa_{S H 9} \\
& A_{1 i j k}=\frac{2 \pi \sqrt{5}}{3 L^{7 / 2}}\left({ }^{i j 1 k} \kappa_{S H 6}\right)-\frac{2 \pi^{3} \sqrt{5}}{3 L^{11 / 2}}\left({ }^{1 i j k} \kappa_{S H 9}+{ }^{i j 1 k} \kappa_{S H 9}\right) \\
& A_{i j k l}=\frac{3 \pi^{4}}{64 L^{3}}{ }^{i j k l} \kappa_{\sigma 4}+\frac{\pi}{16 L}^{i j k l} \kappa_{S H 5}-{\frac{\pi}{16 L^{3}}}^{i j k l} \kappa_{S H 6}-{\frac{\pi}{16 L^{5}}}^{i j k l} \kappa_{S H 9},
\end{aligned}
$$

with $i, j, k, l \geq 2$. It is noted that, in the case of a compressed column, all terms in ${ }^{i j k l}$ $\kappa_{S H 8}$ vanish upon integration.

\subsection{Equilibrium equations}

Differentiating the total potential energy function (27) with respect to the several degrees of freedom yields the equilibrium equations of the system [18]. Specifically, for $i=1$, yields

$$
\frac{\partial A}{\partial^{1} a}=A_{1}+2 A_{11}{ }^{1} a+\sum_{i=2}^{n_{\mathrm{MD}}} \sum_{j=2}^{n_{\mathrm{MD}}}\left(A_{1 i j}{ }^{i} a^{j} a+2 A_{11 i j} a^{i} a^{j} a\right)+\sum_{i=2}^{n_{\mathrm{MD}}} \sum_{j=2}^{n_{\mathrm{MD}}} \sum_{k=2}^{n_{\mathrm{MD}}} A_{1 i j k}{ }^{i} a^{j} a^{k} a=0
$$

while for the remaining degrees of freedom $(i \geq 2)$,

$$
\begin{aligned}
\frac{\partial A}{\partial^{i} a}= & 2 A_{i i}{ }^{i} a+\sum_{\substack{j=2 \\
i \neq j}}^{n_{\mathrm{MD}}}\left(A_{i j}+A_{j i}\right)^{j} a+\sum_{j=2}^{n_{\mathrm{MD}}}\left[\left(A_{1 i j}+A_{1 j i}\right)^{1} a^{j} a+\left(A_{11 i j}+A_{11 j i}\right)^{1} a^{2 j} a\right] \\
& +\sum_{j=2}^{n_{\mathrm{MD}}} \sum_{k=2}^{n_{\mathrm{MD}}}\left[\left(A_{i j k}+A_{j i k}+A_{k j i}\right)^{j} a^{k} a+\left(A_{1 j j k}+A_{1 j i k}+A_{1 k j i}\right)^{1} a^{j} a^{k} a\right] \\
& +\sum_{j=2}^{n_{\mathrm{MD}}} \sum_{k=2}^{n_{\mathrm{MD}}} \sum_{l=2}^{n_{\mathrm{MD}}}\left[\left(A_{i j k l}+A_{j i k l}+A_{k j i l}+A_{l j k i}\right)^{j} a^{k} a^{l} a\right]=0 .
\end{aligned}
$$


Eq. (29) can be solved with respect to ${ }^{1} a$, giving

$$
{ }^{1} a=\frac{-A_{1}-\sum_{i=2}^{n_{\mathrm{MD}}} \sum_{j=2}^{n_{\mathrm{MD}}} A_{1 i j}{ }^{i} a^{j} a-\sum_{i=2}^{n_{\mathrm{MD}}} \sum_{j=2}^{n_{\mathrm{MD}}} \sum_{k=2}^{n_{\mathrm{MD}}} A_{1 i j k}{ }^{i} a^{j} a^{k} a}{2 A_{11}+2 \sum_{i=2}^{n_{\mathrm{MD}}} \sum_{j=2}^{n_{\mathrm{MD}}} A_{11 i j}{ }^{i} a^{j} a}
$$

or, in the case of an open cross-section,

$$
{ }^{1} a=\frac{-A_{1}-\sum_{i=2}^{n_{\mathrm{MD}}} \sum_{j=2}^{n_{\mathrm{MD}}} A_{1 i j}{ }^{i} a^{j} a}{2 A_{11}},
$$

because of the inexistence of a shear flow.

\subsection{Pre-buckling solution and sliding coordinate transformation}

Eqs. (29) and (30) yield a pre-buckling (fundamental) solution defined by

$$
{ }^{1} a_{F}=-\frac{A_{1}}{2 A_{11}}, \quad{ }^{i} a_{F}=0 \text { for } i=2, \ldots, n_{\mathrm{MD}} .
$$

In order to simplify further calculations, it is worth applying a sliding coordinate transformation $[18,19]$

$$
{ }^{i} a={ }^{i} a_{F}+{ }^{i} q, \quad i=1, \ldots, n_{\mathrm{MD}}
$$

to yield a trivial fundamental solution

$$
{ }^{i} q=0, \quad i=1, \ldots, n_{\mathrm{MD}} .
$$

The resulting total potential energy function, now denoted by $W$, is given by

$$
\begin{aligned}
W= & W_{0}+W_{11}{ }^{1} q^{2}+\sum_{i=2}^{n_{\mathrm{MD}}} \sum_{j=2}^{n_{\mathrm{MD}}}\left(W_{i j}{ }^{i} q^{j} q+W_{1 i j}{ }^{1} q^{i} q^{j} q+W_{11 i j}{ }^{1} q^{2 i} q^{j} q\right) \\
& +\sum_{i=2}^{n_{\mathrm{MD}}} \sum_{j=2}^{n_{\mathrm{MD}}} \sum_{k=2}^{n_{\mathrm{MD}}}\left(W_{i j k}{ }^{i} q^{j} q^{k} q+W_{1 i j k}{ }^{1} q^{i} q^{j} q^{k} q\right)+\sum_{i=2}^{n_{\mathrm{MD}}} \sum_{j=2}^{n_{\mathrm{MD}}} \sum_{k=2}^{n_{\mathrm{MD}}} \sum_{l=2}^{n_{\mathrm{MD}}}\left(W_{i j k l}{ }^{i} q^{j} q^{k} q^{l} q\right)
\end{aligned}
$$

where

$$
\begin{aligned}
& W_{0}=-\frac{A_{1}^{2}}{4 A_{11}} \\
& W_{11}=A_{11} \\
& W_{i j}=\left\{\begin{array}{l}
\text { if } i=j, \quad W_{i i}=A_{i i}+A_{11 i i} \frac{A_{1}^{2}}{4 A_{11}^{2}}-A_{1 i i} \frac{A_{i}}{2 A_{11}} \\
\text { if } i \neq j, \quad W_{i j}=A_{i j}+A_{11 i j} \frac{A_{1}^{2}}{4 A_{11}^{2}}-A_{1 i j} \frac{A_{i}}{2 A_{11}}
\end{array}\right.
\end{aligned}
$$




$$
\begin{aligned}
& W_{1 i j}=A_{1 i j}-\frac{A_{11 i j} A_{1}}{A_{11}} \\
& W_{i j k}=A_{i j k}-\frac{A_{1 i j k} A_{1}}{2 A_{11}} \\
& W_{1 i j k}=A_{1 i j k} \\
& W_{11 i j}=A_{11 i j} \\
& W_{i j k l}=A_{i j k l} .
\end{aligned}
$$

\subsection{Linear eigenvalue analysis}

The critical loads of the compressed column are now easily evaluated by setting to zero the determinant of the Hessian matrix of the total potential energy function, evaluated along the fundamental path of the system $[18,19]$ :

$$
\operatorname{det}\left(\boldsymbol{H}_{\boldsymbol{F}}\right)=\left|\frac{\partial^{2} W}{\partial^{i} q \partial^{j} q}\right|_{F}|=| \begin{array}{lllll}
2 W_{11} & 0 & 0 & \cdots & 0 \\
& 2 W_{22} & W_{23}+W_{32} & \cdots & W_{2 n_{\mathrm{MD}}}+W_{n_{\mathrm{MD}} 2} \\
& 2 W_{33} & \cdots & W_{3 n_{\mathrm{MD}}}+W_{n_{\mathrm{MD}} 3} \\
& & \ddots & \vdots \\
& & & 2 W_{n_{\mathrm{MD}} n_{\mathrm{MD}}}
\end{array} \mid=0
$$

Analysing Eq. (38), the following conclusions can be drawn:

(i) the first row and the first column have all terms equal to zero with the exception of the term ${ }^{11} H_{F}$, which is not dependant of $P$; so, when finding the roots of the determinant $\boldsymbol{H}_{\boldsymbol{F}}$, only the submatrix formed by the last $\left(n_{\mathrm{MD}}-1\right)$ rows and columns shall be considered;

(ii) for closed cross-sections, the terms $W_{i j}$, for $i=j$ and $i \neq j$, in the $A_{11 i j}\left(A_{1}^{2} / 4 A_{11}^{2}\right)$ part, contain a quadratic factor in $P$, which is dependent of coefficient ${ }^{11 i j} \kappa_{S H 9}$; so, for closed section members only, if third order coefficients are taken into account for the determination of the critical loads, a non-linear eigenvalue problem will occur [20] - this fact will have a small influence for the value of the critical loads, so in the example presented below, only second order terms will be considered for the closed cross-section;

(iii) for open or closed cross-sections, the terms $W_{i j}$, for $i=j$ and $i \neq j$, contain, in general, a linear term in $P$ in the part $A_{1 i j}\left(A_{i} / 2 A_{11}\right)$, so all coefficients for $i, j \geq 2$ will have a constant part and a linear part in $P$ - thus, the eigenvalue problem is established.

Regarding the first conclusion above, the eigenvalue-eigenvector problem will have dimension $\left(n_{\mathrm{MD}}-1\right)$, since coordinate ${ }^{1} q$ will always be passive. From the third conclusion, the shape of any term $(i, j)$ of $\boldsymbol{H}_{\boldsymbol{F}}$, for any $i, j \geq 2$, is ${ }^{i j} H_{0}+{ }^{i j} H_{1} P$, 
and the generalized eigenvalue problem can be established in the form [21]:

$$
\left(\boldsymbol{H}_{0}+P \cdot \boldsymbol{H}_{1}\right) \boldsymbol{q}=0,
$$

being the non-zero terms in the eigenvectors $\boldsymbol{q}$, if properly normalized, regarded as the "participation" of their relative mode of deformation in the overall buckling note that in this particular case, each coordinate ${ }^{i} q$ is related to a specific mode of deformation.

\section{Example}

In order to illustrate the use of the energy formulation in the context of GBT, the stability analysis of a compressed thin-walled prismatic member with open or closed cross-section is presented. The closed cross-section - case 1 -consists of a RHS $80 \times 40 \times 2$ member while the open section - case 2 -was chosen to have the same height, width and cross-sectional area as and also to fulfil EC3 - part 1.3 recommendations for lip slenderness [22]. Fig. 5 illustrates the cross-section geometry and the nodal discretization for the chosen examples. The Young modulus is $E=210 \mathrm{GPa}$ and the Poisson ratio is $\mu=0.3$. The warping functions, the cross-section displacements and the transverse bending moments are shown in Figs. 6-8. For a compressive axial force $P$ applied at one member end (see Fig. 9), the potential of the external load is simply given by:

$$
\Pi_{P, 1}=P \times\left.{ }^{1} V^{\prime}\right|_{x=L}={ }^{1} a \frac{2 \sqrt{5}}{L^{3 / 2}} P .
$$

Being both cases related to equal cross-section areas - that is equivalent to say, in GBT notation, to equal values of ${ }^{1} C$ [11] - their fundamental paths become

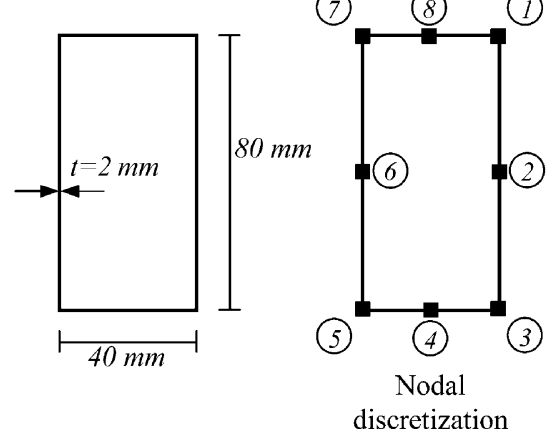

Case 1
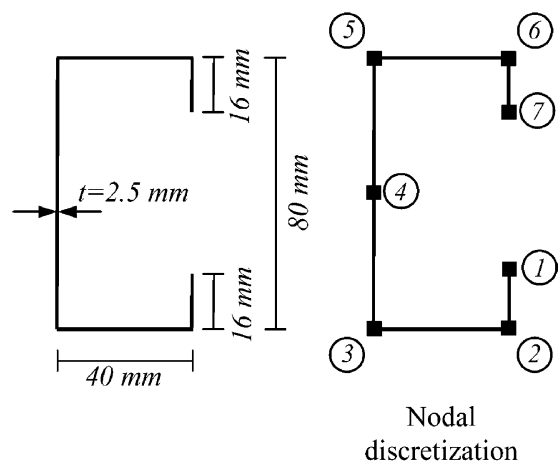

Fig. 5. Definition of cross-section dimensions and nodal discretization. 


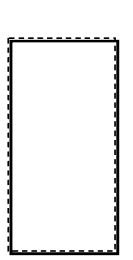

Mode 1

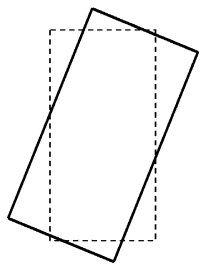

Mode
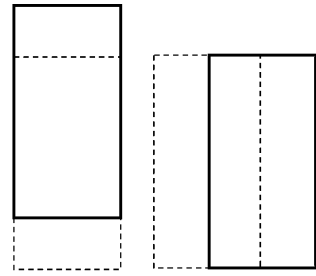

Mode 2 Mode 3

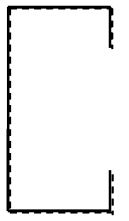

Mode 1

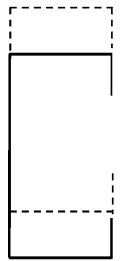

Mode 2

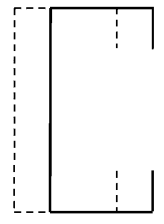

Mode 3

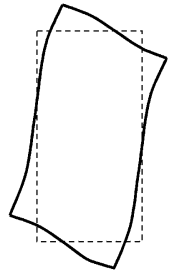

Mode 5

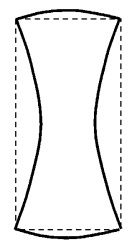

Mode 6

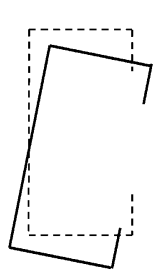

Mode 4

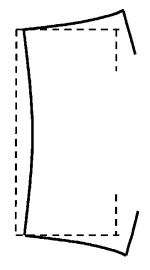

Mode 5

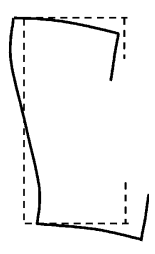

Mode 6

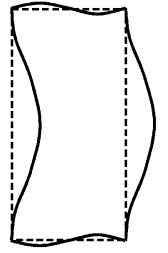

Mode 7

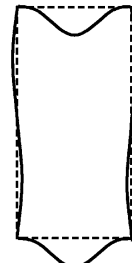

Mode 8

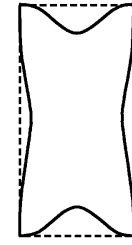

Mode 9

Case 1

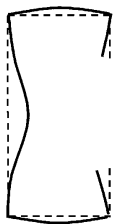

Mode 7

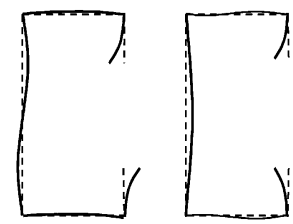

Mode 8 Mode 9

Case 2

Fig. 6. Shape of the modal cross-section displacements.

equal and given by:

$$
{ }^{1} a_{1}=2.21832 \times 10^{-6} \times L^{5 / 2},
$$

all other coordinates being equal to zero. Applying standard stability procedures $[18,19]$, the critical loads presented in Fig. 10 are obtained, together with the modal participation coefficients.

In case 1, the existence of three buckling regions is clearly perceptible. The first region, where modes 6 and 5 are predominant, corresponds to the local plate buckling zone, for lengths smaller than $400 \mathrm{~mm}$. Then, as the member length increases, the second region occurs, where modes 3 and 7 interact, this phenomenon being related to global buckling-minor axis bending-influenced by buckling of the compressed web, linked to the asymmetric distortional mode. Finally, for lengths greater than about $1500 \mathrm{~mm}$, the third region occurs, where global buckling mode (minor axis bending) alone governs the behaviour of the member. It is important 

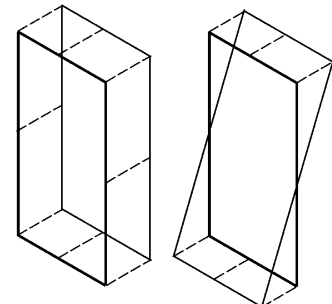

Mode 1

Mode 2

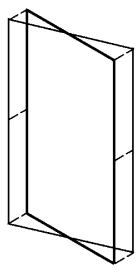

Mode 3

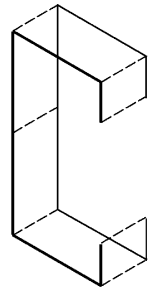

Mode 1

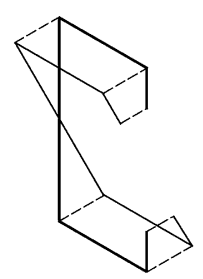

Mode 2

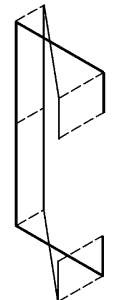

Mode 3

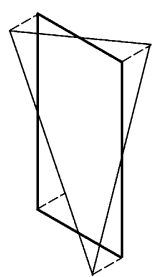

Mode 4

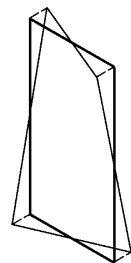

Mode 5

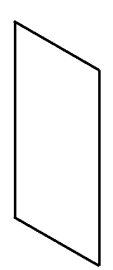

Mode 6

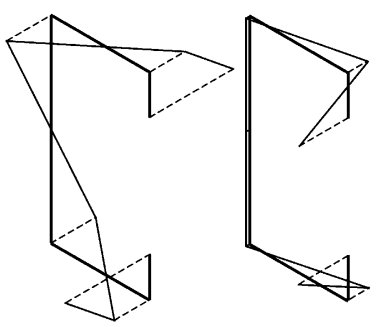

Mode 4

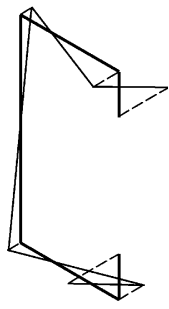

Mode 6

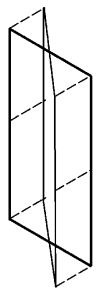

Mode 7

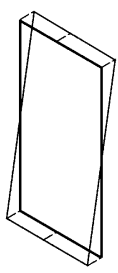

Mode 8

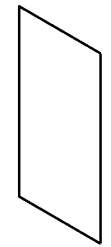

Mode 9

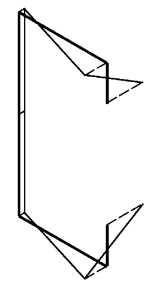

Mode 7

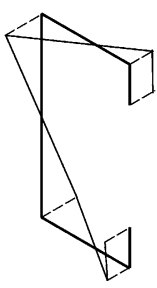

Mode 8

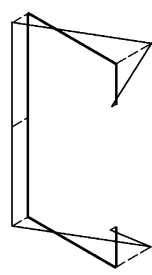

Mode 9

\section{Case 1}

Case 2

Fig. 7. Shape of the modal warping displacements.

to note that individual mode buckling loads for modes 4 and 5 are much greater than the buckling loads for all modes, as can be observed in Fig. 11.

The buckling behaviour and the modal participation for case 2 are more complex, due to the fact that this case deals with a monosymmetric open cross-section. Four buckling regions can be identified. The first region is related to lengths smaller than $100 \mathrm{~mm}$ and local plate buckling rules the column behaviour, with a small influence of the lips buckling. In GBT notation, this fact is expressed by the influence of mode 6 coupled with mode 9 . Then, a second region occurs for lengths up to $530 \mathrm{~mm}$ and mode 5 rules the buckling loads, corresponding to the symmetric distortional buckling zone. In the third region, for column lengths between 530 and $2100 \mathrm{~mm}$, body modes 2, 4 and 6 rule the behaviour - in this zone, at first, mode 6 (asymmetric distortional mode) governs the behaviour but smoothly major 


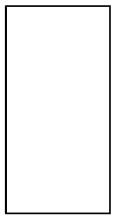

Mode 1

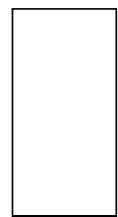

Mode 2

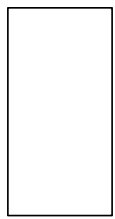

Mode 3

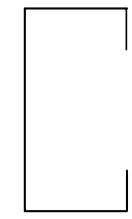

Mode 1

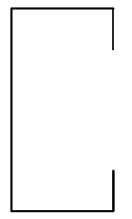

Mode 2 Mode 3

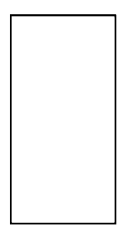

Mode 4

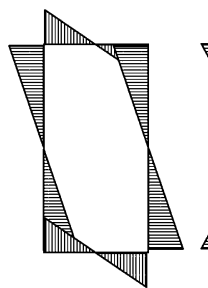

Mode 5

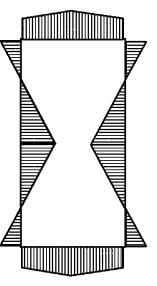

Mode 6

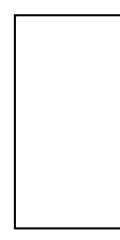

Mode 4

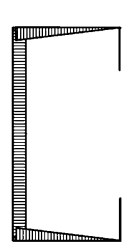

Mode 5

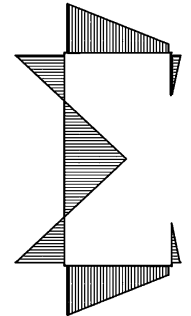

Mode 7

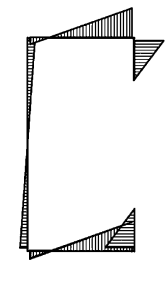

Mode 8

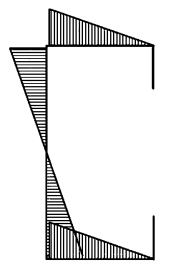

Mode 6

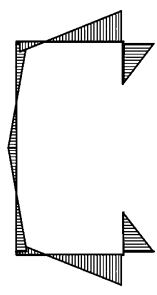

Mode 9

Case 1

Fig. 8. Shape of the modal transverse bending diagrams.

axis bending (mode 2) takes the most relevant role. Then, for lengths higher than $2100 \mathrm{~mm}$, minor axis bending starts suddenly to rule alone the behaviour of the column.

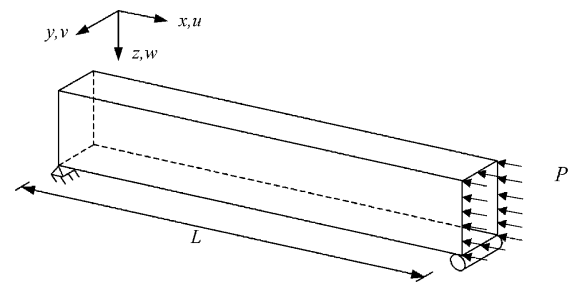

Case 1

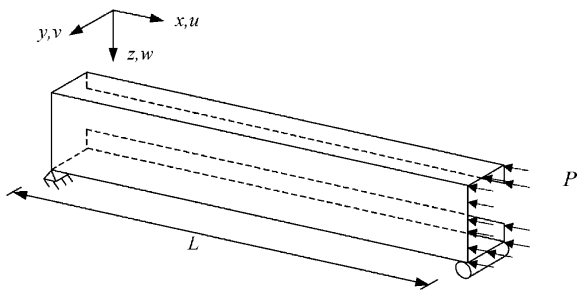

Case 2

Fig. 9. The compressed column. 

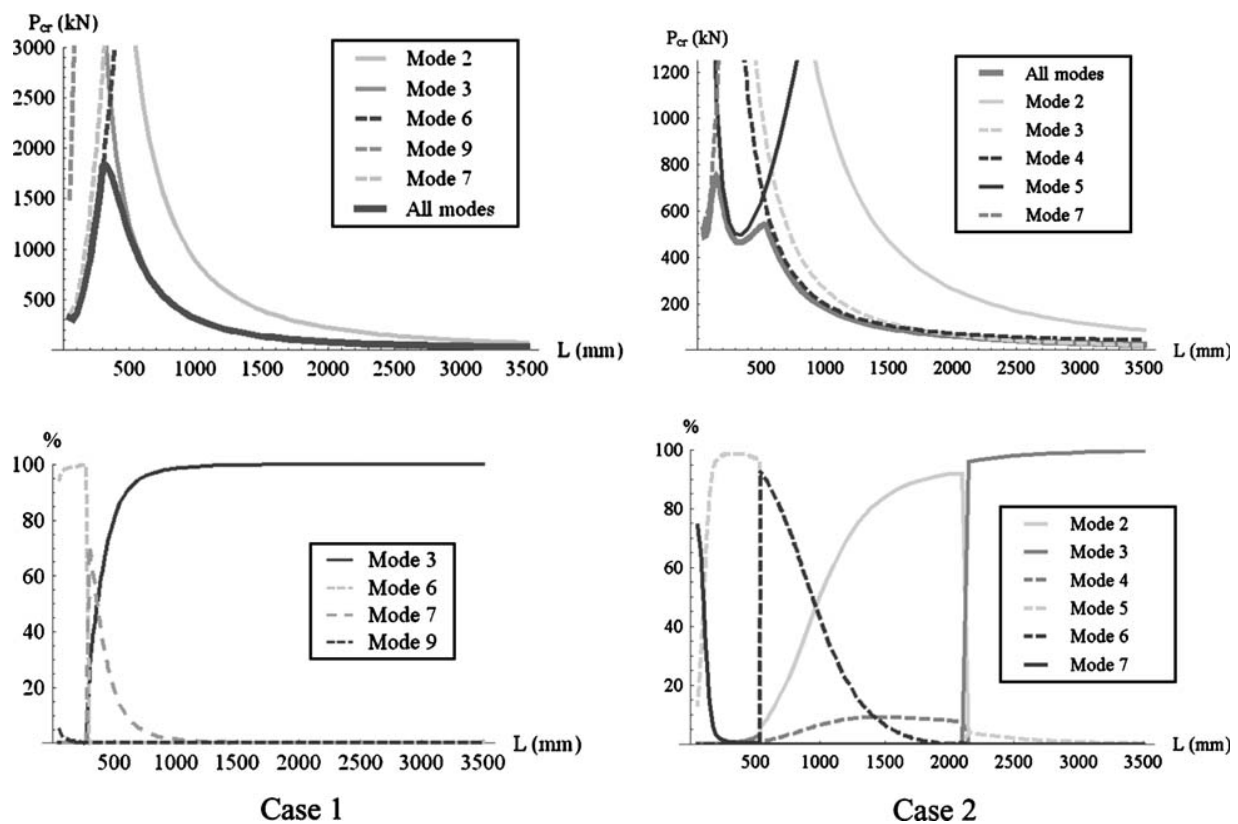

Fig. 10. Buckling loads and modal participations for cases 1 and 2.

Observing Fig. 12, it can be concluded that the buckling loads for the closed cross-section are much greater for a lengths range between 250 and $1600 \mathrm{~mm}$ (in the open section case, this lengths range corresponds to the 2 nd and $3 \mathrm{rd}$ regions) because, for these lengths, symmetric and asymmetric distortional buckling rule the open section buckling loads behaviour and they are not present or not relevant for the closed section case. However, the thickness of the open section is greater and this fact implies a greater buckling load only for very small lengths, for those

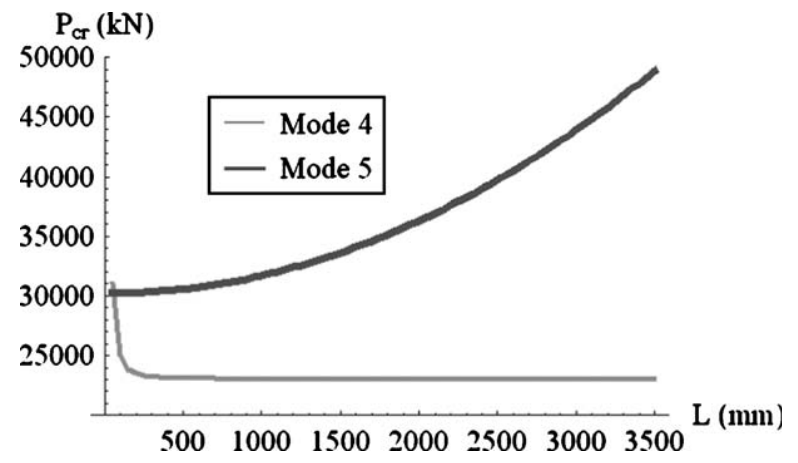

Fig. 11. Single mode buckling loads for modes 4 and 5 for case 1. 


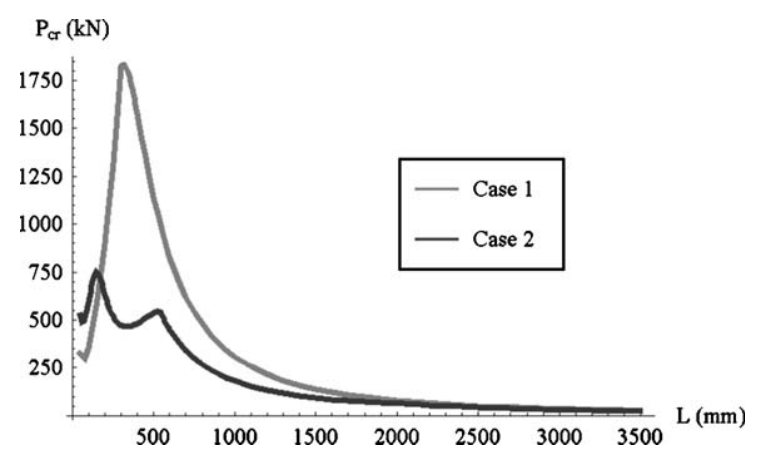

Fig. 12. All modes bifurcation loads for cases 1 and 2.

where local plate buckling is the most relevant buckling mode for the open and for the closed sections.

\section{Conclusions}

A unified energy formulation for the analysis of the stability of open or closed thin-walled cross-section members in the framework of GBT was presented. This formulation highlights the specific differences between the two types of sections, namely the presence of a shear flow for closed cross-section members and the need to take into account the membrane shear distortion. Using a Rayleigh-Ritz approach and sinusoidal approximations for the various modes of deformation, it was possible to obtain solutions for the various bifurcation loads. Application to the evaluation of the stability of a compressed column for two different cross-section alternatives with constant area has shown a net gain in the buckling resistance for the closed cross-section elements for the intermediate slenderness range.

Finally, the energy formulation is able to deal with the post-buckling behaviour of thin-walled members, since it already includes the relevant non-linear terms, an issue being currently actively pursued.

\section{Acknowledgements}

Financial support from "PRODEP-Acção de Formação Avançada de Docentes do Ensino Superior (Ministério da Educação)" for Pedro Simão is gratefully acknowledged.

\section{References}

[1] Davies JM. Recent research advances in cold-formed steel structures. Journal of Constructional Steel Research 2000;55:267-88.

[2] Yu W-W. Cold-formed steel design. New York: John Wiley \& Sons; 2000. 
[3] Cheung YK. Finite strip method in structural analysis. Pergamon Press; 1976.

[4] Schardt R. Generalized beam theory — an adequate method for coupled stability problems. ThinWalled Structures 1994;19:161-80.

[5] von Kármán T, Christensen NB. Methods of analysis for torsion with variable twist. Journal of the Aeronautical Sciences (II) 1944;April:110-24.

[6] Vlasov VZ. Thin walled elastic beams, 2nd ed. Washington: The National Science Foundation, Department of Commerce; 1961.

[7] Schardt R. Eine Erweiterung der Technischen Biegetheorie zur Berechnung prismatischer Faltwerke. Der Stahlbau 1966;35:161-71.

[8] Schardt R. Anwendung der Erweiterten Technischen Biegetheorie auf die Berechnung prismatischer Faltwerke und Zylinderschalen nach Theorie I. und II. Ordnung. Proceedings of the IASS-Symposium on Folded Plates and Prismatic Structures, Wien, vol. I. 1970.

[9] Miosga G. Vorwiegend längsbeanspruchte dünnwandige prismatische Stäbe und Platten mit endlichen elastischen Verformungen. Dissertation D 17. Technische Hochschule Darmstadt, 1976.

[10] Möller R. Zur Berechnung prismatischer Strukturen mit beliebigem nicht formtreuem Querschnitt, Bericht N. 2 des Institut für Statik der TH Darmstadt. Technische Hochschule Darmstadt, 1982.

[11] Schardt R. Verallgemeinerte Technische Biegetheorie. Berlin, Heidelberg: Springer Verlag; 1989.

[12] Davies JM, Leach P, Heinz D. Second-order generalised beam theory. Journal of Constructional Steel Research 1994;31(2-3):221-41.

[13] Davies JM, Jiang C, Ungureanu V. Buckling mode interaction in cold formed steel columns and beams. Proceedings of the 14th International Specialty Conference on Cold Formed Steel Structures, St. Louis, Missouri, USA, October 15-16. University of Missouri-Rolla. 1998, p. 53-67.

[14] Born J. Faltwerke-Ihre Theorie und Berechnung. Stuttgart: Verlag Konrad Wittwer; 1954.

[15] Girkmann K. Flächentragwerke. Wien: Springer-Verlag; 1959.

[16] Kolbrunner CF, Hajdin N. Dünnwandige Stäbe-Band 2: Stäbe mit deformierbaren Querschnitten, Nicht-elastisches Verhalten dünnwandiger Stäbe. Berlin, Heidelberg: Springer-Verlag; 1975.

[17] Richards TH. Energy methods in stress analysis. Chichester: Ellis Horwood; 1977.

[18] Thompson JMT, Hunt G. A general theory of elastic stability. John Wiley \& Sons; 1973.

[19] Thompson JMT, Hunt G. Elastic instability phenomena. John Wiley \& Sons; 1984.

[20] Mirasso AE, Godoy LA. Iterative techniques for non-linear eigenvalue buckling problems. Communications in Applied Numerical Methods 1992;8:311-7.

[21] Hangai Y, Kawamata S. Perturbation method in the analysis of geometrically nonlinear and stability problems. In: Oden JT, Clough RW, Yamamoto Y, editors. Advances in computational methods in structural mechanics and design. Huntsville (AL), USA: UAH Press; 1972, p. 473-89.

[22] European Committee for Standardization. EC 3-Eurocode 3, Design of steel structures, part 1.3general rules - supplementary rules for cold formed thin gauge members and sheeting. 1996. 